\title{
Domain Sorting in Giant Unilamellar Vesicles Adsorbed on Glass
}

\author{
Chiho Kataoka-Hamai* and Kohsaku Kawakami \\ Research Center for Functional Materials, National Institute for Materials Science \\ 1-1 Namiki, Tsukuba, Ibaraki 305-0044, Japan \\ *E-mail: kataoka.chiho@nims.go.jp
}

Number of pages: 1

\section{SUPPORTING INFORMATION - MOVIE LEGEND}

\section{Movie 1.avi}

Movie of the loosely adsorbed GUV observed at $0-7.887 \mathrm{~s}$ in Figure 3B. The image size is $11 \times 11 \mu \mathrm{m}^{2}$. Each frame was acquired in $51 \mathrm{~ms}$. The movie was constructed using frames every 3 steps. Playback speed is 10 frames per second (53 frames total).

\section{Movie 2.avi}

Movie of the $L_{d}$ domain sorting in the GUV recorded at $29 \min 38.222-42.216 \mathrm{~s}$ in Figure 3B. The image size is $16 \times 16 \mu \mathrm{m}^{2}$. Each frame was acquired over $51 \mathrm{~ms}$. Playback speed is 10 frames per second $(80$ frames total).

\section{Movie 3.avi}

Movie of the GUV in Figure 3B. The ring-like $\mathrm{L}_{\mathrm{o}}$ domain growth and rupture were recorded at $29 \mathrm{~min}$ $54.508-58.148 \mathrm{~s}$. The image size is $28 \times 30 \mu \mathrm{m}^{2}$. Each frame was acquired over $51 \mathrm{~ms}$. Playback speed is 10 frames per second (73 frames total)

\section{Movie 4.avi}

Movie of the loosely adsorbed GUV observed at $0-19.975 \mathrm{~s}$ in Figure 3D. The image size is $13 \times 13 \mu \mathrm{m}^{2}$. Each frame was acquired in $51 \mathrm{~ms}$. The movie was constructed using frames every 5 steps. Playback speed is 10 frames per second ( 80 frames total). 